\title{
Multimodal perioperative pain protocol for Gynecologic Oncology laparotomy reduces length of hospital stay
}

\author{
Jordan N. Mattson, MD, ${ }^{1}$ MacKenzie Thayer, BS, ${ }^{2}$ Sarah L. Mott, MS, ${ }^{3}$ Yasmin A. \\ Lyons, DO, ${ }^{1}$ Henry D. Reyes, MD ${ }^{1}$ Megan E. McDonald, MD ${ }^{1}$ Abbey J. Hardy- \\ Fairbanks, MD, ${ }^{1}$ Emily K. Hill, $\mathrm{MD}^{1,3}$
}

Keywords: Post-operative care, multimodal pain regimen, gynecologic oncology, laparotomy, perioperative care, enhanced recovery after surgery

Objectives: Our primary objective was to evaluate the impact of a multimodal perioperative pain regimen on length of hospital stay for patients undergoing laparotomy with a gynecologic oncologist.

Methods: We compared 52 patients who underwent laparotomy with a gynecologic oncologist at a single institution in 2017-2018 after implementation of a multimodal perioperative pain regimen to a historic cohort of 94 patients (2016-2017). The multimodal pain regimen included pre and post-operative administration of oral acetaminophen, gabapentin, and celecoxib, in addition to standard narcotics and optional epidural analgesia. Demographic, surgical, and post-operative data were collected. Linear regression models were used to determine factors associated with length of stay.

Results: On multivariable analysis, bowel resection, stage, surgery length, age and group (pre vs. post pain protocol implementation) were retained as significant independent predictors of length of stay. Patients undergoing gynecologic oncology laparotomy prior

\footnotetext{
${ }^{1}$ Department of Obstetrics and Gynecology, University of lowa Carver College of Medicine, lowa City, IA

${ }^{2}$ Carver College of Medicine, University of lowa Hospitals and Clinics, Iowa City, IA, 52242

${ }^{3}$ Holden Comprehensive Cancer Center, University of lowa Hospitals and Clinics, lowa City, IA
}

Please cite this as: Mattson JN, Thayer M, Mott SL, Lyons YA, Reyes HD, McDonald ME, HardyFairbanks AJ, Hill EK. Multimodal perioperative pain protocol for Gynecologic Oncology laparotomy reduces length of hospital stay. Proc Obstet Gynecol. 2018;8(3):Article 25 [ 2 p.]. Available from: http://ir.uiowa.edu/pog/. Free full text article.

Corresponding author: Jordan N. Mattson, MD, Department of Obstetrics and Gynecology, 200 Hawkins Drive, lowa City, IA 52246. Email: jordan-mattson@uiowa.edu

Copyright: (c) 2018 Mattson et al. This is an open-access article distributed under the terms of the Creative Commons Attribution License, which permits unrestricted use, distribution, and reproduction in any medium, provided the original author and source are credited. 
to the implementation of the pain protocol had a length of stay 1.26 times longer than patients undergoing laparotomy during the postimplementation period $(p<0.01)$. For the more complex surgical patients who received the multimodal pain regimen (those with stage II-IV disease who underwent bowel resection), this translated into a reduction in length of hospital stay of 1.73 days when compared to the matched cohort. There was a significant reduction in average pain scale score on post-operative day zero from 4.49 to $3.63(\mathrm{p}=0.02)$ and an overall reduction of morphine equivalents used between the two groups on post-operative days $0-2$, although this did not reach statistical significance. Adverse outcomes between groups, including ileus, delirium, renal failure and re-admission, were similar.

Conclusions: Implementation of a multimodal perioperative pain regimen in patients undergoing gynecologic oncology laparotomy was associated with a significant reduction of length of hospital stay and improved pain scores without increased complications. Even in the absence of a complete Enhanced Recovery in Surgery Protocol, a multimodal perioperative pain regimen has the potential to shorten hospital stay and improve patient perceived pain.

Learning Objectives: Implementation of a multimodal perioperative pain regimen in gynecologic oncology laparotomy patients has been shown to reduce length of post-operative hospital stay and decrease patient perceived pain on POD 0.

Presented at "Complicated Maternal Fetal Medicine Cases," the University of lowa Carver College of Medicine Ob/Gyn Postgraduate Conference, 2 November 2018, Hilton Garden Inn, lowa City, lowa. 